CRYSTALLOGRAPHIC COMMUNICATIONS

ISSN 2056-9890

Reçu le 25 août 2017

Accepté le 4 septembre 2017

Edité par L. Van Meervelt, Katholieke Universiteit Leuven, Belgique

Keywords: perchlorate; organic-inorganic hybrid; crystal structure; graph-set motifs.

CCDC reference: 1010810

Supporting information: this article has supporting information at journals.iucr.org/e

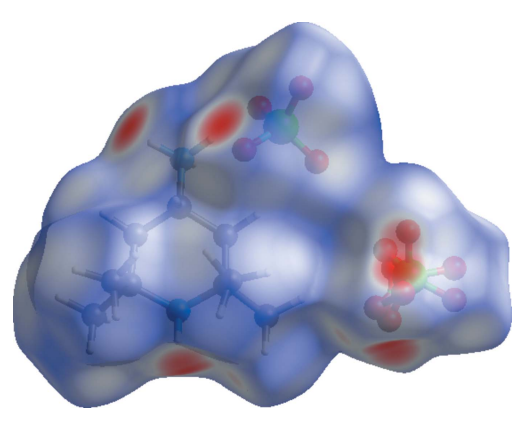

OPEN $\odot$ ACCESS

\section{Structure cristalline et analyses thermique et de surface Hirshfeld du diperchlorate de 4-azaniumyl- 2,2,6,6-tétraméthylpipéridin-1-ium}

\author{
Hammouda Chebbi, ${ }^{a, b *}$ Abdessalem Boumakhla, ${ }^{a}$ Mohamed Faouzi Zid ${ }^{\mathrm{a}}$ et \\ Abderrahmen Guesmi ${ }^{\mathrm{a}}$
}

\begin{abstract}
a Université de Tunis El Manar, Faculté des Sciences de Tunis, Laboratoire de Matériaux, Cristallochimie et Thermodynamique Appliquée, 2092 El Manar II, Tunis, Tunisia, et ${ }^{\mathbf{b}}$ Institut Préparatoire aux, Etudes d'Ingénieurs de Tunis, rue Jawaher Lel Nehru, 1089 Montfleury, Tunis, Tunisia. *Correspondence e-mail: chebhamouda@yahoo.fr
\end{abstract}

The synthesis of 4-azaniumyl-2,2,6,6-tetramethylpiperidin-1-ium diperchlorate, $\mathrm{C}_{9} \mathrm{H}_{22} \mathrm{~N}_{2}{ }^{2+} \cdot 2 \mathrm{ClO}_{4}{ }^{-}$, was carried out from an aqueous reaction of perchloric acid with 4-amino-2,2,6,6-tetramethylpiperidine. This compound was characterized by TGA-DSC analysis and single-crystal X-ray diffraction. The piperidine ring of the dication adopts a chair conformation and the orientation of the $\mathrm{C}-\mathrm{NH}_{3}$ bond is equatorial. One of the two crystallographically independent perchlorate anions exhibits disorder [occupancies $0.625(7)$ and $0.375(7)$ ]. The crystal packing is constituted by a succession of mixed layers parallel to the (102) plane, made up of $\mathrm{C}_{9} \mathrm{H}_{22} \mathrm{~N}_{2}{ }^{2+}$ dications and $\mathrm{ClO}_{4}{ }^{-}$anions. These ions are linked by normal and bifurcated $\mathrm{N}-\mathrm{H} \cdots \mathrm{O}$ hydrogen bonds with $R_{4}^{4}(12)$ graph-set motifs, generating a two-dimensional network. The intermolecular interactions in the crystal structure were quantified and analysed using Hirshfeld surface analysis.

\section{Contexte chimique}

Les matériaux hybrides 'organique-inorganique' sont l'objet d'un intérêt sans cesse croissant permettant d'allier à la fois certaines propriétés d'un matériau inorganique (ou d'une molécule inorganique), et certaines propriétés d'un polymère (ou d'une molécule organique). Cette symbiose entre deux mondes de la chimie trop longtemps considérés comme antagonistes peut aussi amener à des propriétés complètement nouvelles, et ouvre un vaste champ d'investigations pour le chimiste.

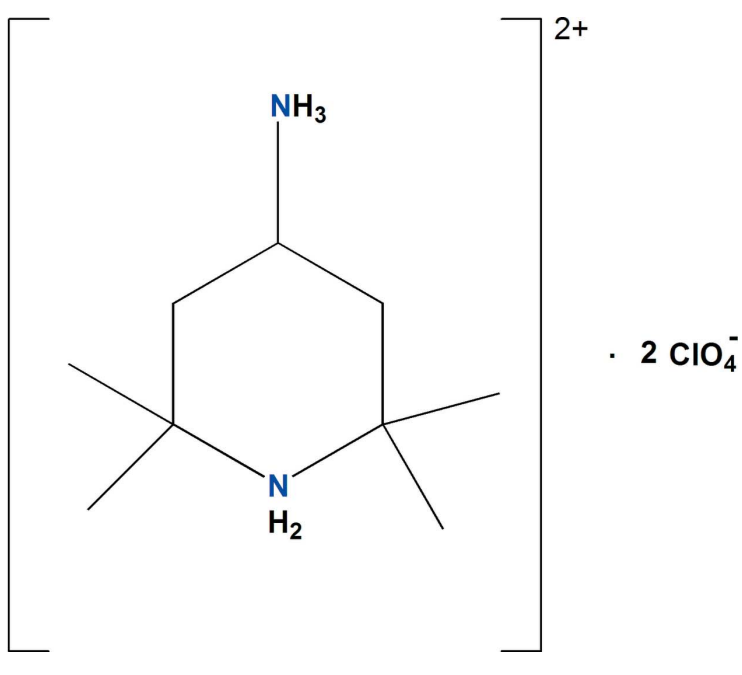




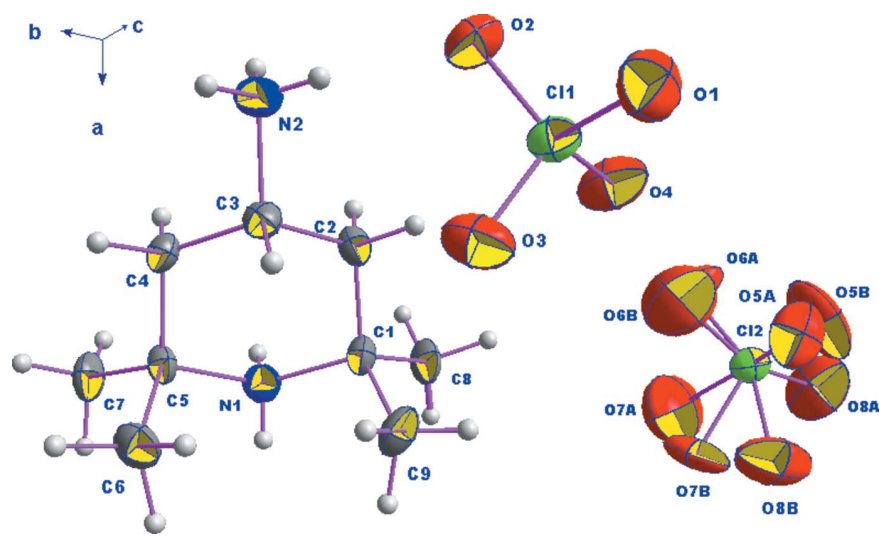

Figure 1

L'unité asymétrique de (I). Les ellipsoïdes d'agitation thermique ont $30 \%$ de probabilité d'existence.

Les applications de ces matériaux hybrides couvrent des champs variés telle que les propriétés de résistance mécanique (Mammeri et al., 2005) et le doublage de fréquence (Mackenzie, 1993) d'où leurs applications dans le domaine de l'électronique. De leur côté, les composés hybrides à base de perchlorates ont été particulièrement étudiés du fait des propriétés physiques intéressantes qu'ils présentent comme (1) la ferroélectricité: perchlorate de pyridinium (Czamecki et al., 1994), perchlorate de pyridin-4-ylméthanaminium (Cui et al., 2016), perchlorate de guanidinium (Drozd \& Dudzic, 2013) et (2) l'optique non-linéaire: perchlorate d'anilinium (Vivek et al., 2015), perchlorate de $p$-nitroanilinium (Bouchouit et al., 2008), perchlorate de L-leucinium (Marchewka \& Drozd, 2013). La condition fondamentale pour le test de l'optique non-linéaire est que le composé soit non centrosymétrique.

Dans la chimie des perchlorates et en particulier les perchlorates à cations organiques, plusieurs structures décrites dans la littérature (Dai, 2008; Zhang, 2009; Anitha et al., 2013; Direm et al., 2015) présentent une cristallochimie particulière avec des liaisons hydrogène assurant leurs cohésions.

Ce travail porte sur un perchlorate à cation organique qui résulte de l'interaction entre l'acide perchlorique et une molécule organique possédant deux doublets électroniques libres: 4-amino-2,2,6,6-tétraméthylpipéridine. Il s'agit du composé $\left(\mathrm{C}_{9} \mathrm{H}_{22} \mathrm{~N}_{2}\right)\left[\mathrm{ClO}_{4}\right]_{2}(\mathrm{I})$. Nous présentons ici sa structure cristalline et ses caractérsitiques thermique (TG-DSC) ainsi que l'analyse de sa surface Hirshfeld.

\section{Analyse structurale}

La détermination structurale du composé diperchlorate de 4-azaniumyl-2,2,6,6-tétraméthylpipéridin-1-ium de formule $\left(\mathrm{C}_{9} \mathrm{H}_{22} \mathrm{~N}_{2}\right)\left[\mathrm{ClO}_{4}\right]_{2}(\mathrm{I})$, a permis d'établir le modèle structural, dont l'unité asymétrique est constituée d'un cation organique diprotoné et de deux anions perchlorate dont un est désordonné (Fig. 1). La jonction entre ces entités est assurée par des liaisons hydrogène modérées de type $\mathrm{N}-\mathrm{H}$...O (Jeffrey, 1997). La structure cristalline de (I) est constituée par une succession de couches mixtes formées d'anions et de cations, parallèles aux plans (102) (Fig. 2).

Dans le composé étudié un des anions, $\mathrm{Cl}_{2} \mathrm{O}_{4}{ }^{-}$, présente un désordre qui se manifeste par l'agitation thermique de tous les atomes d'oxygène. Ainsi, chaque atome d'oxygène se trouve partagé entre deux sites cristallographiques avec des taux d'occupation de 0,625 (7) et 0,375 (7). Une recherche bibliographique sur les composés perchloratés a révélée l'existence de quatre composés similaires présentant le même phénomène de désordre (Butcher et al., 2002; Sutha Siluvai et al., 2005; Kaabi et al., 2010, 2011). L'environnement de l'autre atome de chlore $\mathrm{Cl} 1$ est tétraédrique avec une déformation considérable. En effet, le tétraèdre $\mathrm{Cl1O}_{4}$ contient deux liaisons $\mathrm{Cl}-\mathrm{O}$ courtes qui valent respectivement 1,413 (3) et 1,422 (3) $\AA$ et deux autres plus longues $1,433(3)$ et $1,438(3) \AA$. Les distances les plus longues concernent les deux atomes d'oxygène $\mathrm{O} 2$ et $\mathrm{O} 3$ engagés dans les liaisons hydrogène. Ces valeurs sont comparables à celles du même anion étudié avec d'autres types de cations (Bendjeddou et al., 2003; Avecilla et al., 2005; Zhao, 2012; Gholizadeh et al., 2014).

Les cations organiques $\left(\mathrm{C}_{9} \mathrm{H}_{22} \mathrm{~N}_{2}\right)^{2+}$ occupent des positions générales et assurent l'équilibre des charges négatives excédentaires portées par les anions $\mathrm{ClO}_{4}{ }^{-}$et contribuent à la cohésion structurale. Le cycle pipéridine de ce cation adopte la conformation chaise avec orientation équatoriale pour la liaison $\mathrm{C}-\mathrm{NH}_{3}{ }^{+}$. Dans le groupement organique, les valeurs des longueurs de liaison $\mathrm{C}-\mathrm{C}$ varient de 1,522 (5) à 1,539 (5) $\AA$; la valeur moyenne des distances $\mathrm{C}-\mathrm{N}$ est égale à 1,525 (4) $\AA$ et les angles $\mathrm{C}-\mathrm{C}-\mathrm{C}, \mathrm{C}-\mathrm{C}-\mathrm{N}, \mathrm{C}-\mathrm{N}-\mathrm{C}$ sont compris entre $105,5(3)$ et $120,7(2)^{\circ}$. Ces valeurs ne présentent

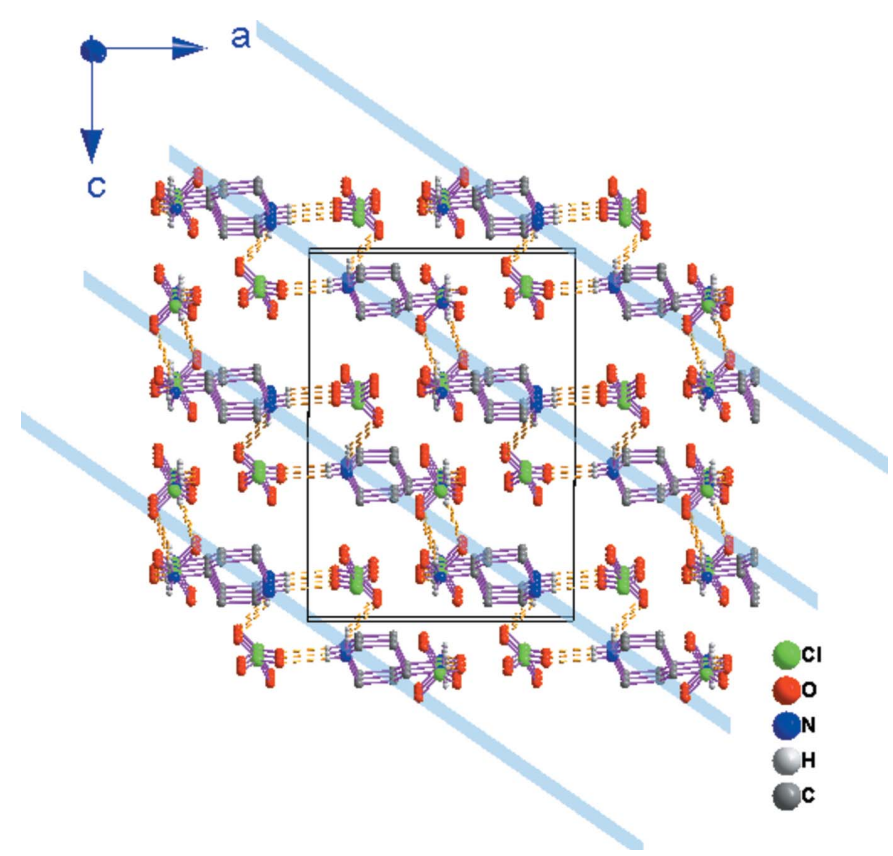

Figure 2

Vue en perspective de la structure bidimensionnelle du composé (I) montrant les couches mixtes parallèles au plan $(\overline{1} 02)$. Pour la clarté de la figure on a representé uniquement les atomes d'oxygène possédant le taux d'occupation le plus élevé dans le perchlorate désordonné et les atomes d'hydrogène des groupements $-\mathrm{NH}_{2}{ }^{+}$et $-\mathrm{NH}_{3}{ }^{+}$. 
Tableau 1

Géométrie des liaisons hydrogène $\left(\AA,^{\circ}\right)$.

\begin{tabular}{lllll}
\hline$D-\mathrm{H} \cdots A$ & $D-\mathrm{H}$ & $\mathrm{H} \cdots A$ & $D \cdots A$ & $D-\mathrm{H} \cdots A$ \\
\hline $\mathrm{N} 1-\mathrm{H} 1 B \cdots \mathrm{O} 5 A^{\mathrm{i}}$ & $0,84(4)$ & $2,38(4)$ & $3,178(7)$ & $158(3)$ \\
$\mathrm{N} 1-\mathrm{H} 1 B \cdots \mathrm{O} 8 B^{\mathrm{i}}$ & $0,84(4)$ & $2,19(5)$ & $2,975(12)$ & $155(3)$ \\
$\mathrm{N} 1-\mathrm{H} 1 A \cdots \mathrm{O} 7 B^{\mathrm{ii}}$ & $0,91(3)$ & $2,06(4)$ & $2,885(9)$ & $150(3)$ \\
$\mathrm{N} 1-\mathrm{H} 1 A \cdots \mathrm{O} 7 A^{\mathrm{ii}}$ & $0,91(3)$ & $2,17(4)$ & $3,069(14)$ & $169(3)$ \\
$\mathrm{N} 1-\mathrm{H} 1 A \cdots \mathrm{O} 8 B^{\mathrm{ii}}$ & $0,91(3)$ & $2,35(4)$ & $3,086(13)$ & $138(3)$ \\
$\mathrm{N} 2-\mathrm{H} 2 A \cdots \mathrm{O} 2$ & $0,87(8)$ & $2,09(8)$ & $2,947(6)$ & $168(6)$ \\
$\mathrm{N} 2-\mathrm{H} 2 B \cdots \mathrm{O}{ }^{\mathrm{iii}}$ & $0,92(6)$ & $2,04(6)$ & $2,951(6)$ & $171(5)$ \\
\hline
\end{tabular}

Codes de symétrie: (i) $x, y+1, z$; (ii) $-x+2,-y+1,-z$; (iii) $-x+1, y+\frac{1}{2},-z-\frac{1}{2}$.

pas d'anomalies, elles sont comparables aux structures contenant le même cation 4-amino-2,2,6,6-tétraméthylpipéridine (Huang \& Deng, 2007; Mrad et al., 2009; Chebbi \& Driss, 2001; Chebbi et al., 2014).

\section{Caractéristiques supramoléculaires}

La cohésion de l'édifice cristallin est assurée principalement par sept liaisons hydrogène modérées de type $\mathrm{N}-\mathrm{H}$ - . O entre les cations et les anions (Jeffrey, 1997). Les distances et les angles décrivant les liaisons hydrogène sont donnés dans le Tableau 1.

Les anions $\mathrm{ClO}_{4}{ }^{-}$jouent un rôle important dans la cohésion de la structure du fait qu'ils participent dans les liaisons hydrogène de type $\mathrm{N}-\mathrm{H} \cdots \mathrm{O}$ en acceptant des atomes d'hydrogène de la partie organique. En effet, les tétraèdres $\mathrm{Cl}_{1} \mathrm{O}_{4}$ se connectent avec les groupements $-\mathrm{N}_{2} \mathrm{H}_{3}{ }^{+}$du dication organique pour former des chaînes ondulées se developant selon la direction [201] donnant lieu aux motifs de liaisons hydrogène $R_{4}^{4}(12)$ (Fig. 3). Dans une couche mixte les cations $\left(\mathrm{C}_{9} \mathrm{H}_{22} \mathrm{~N}_{2}\right)^{2+}$ orientent leurs groupements $-\mathrm{NH}_{2}{ }^{+}$et $-\mathrm{NH}_{3}{ }^{+}$ vers les atomes d'oxygène des anions perchlorate permettant ainsi d'établir des liaisons hydrogène de type $\mathrm{N}-\mathrm{H} \cdots \mathrm{O}$, intraet inter-chaînes, qui contribuent à la cohésion bidimensionnelle du réseau (Fig. 4).

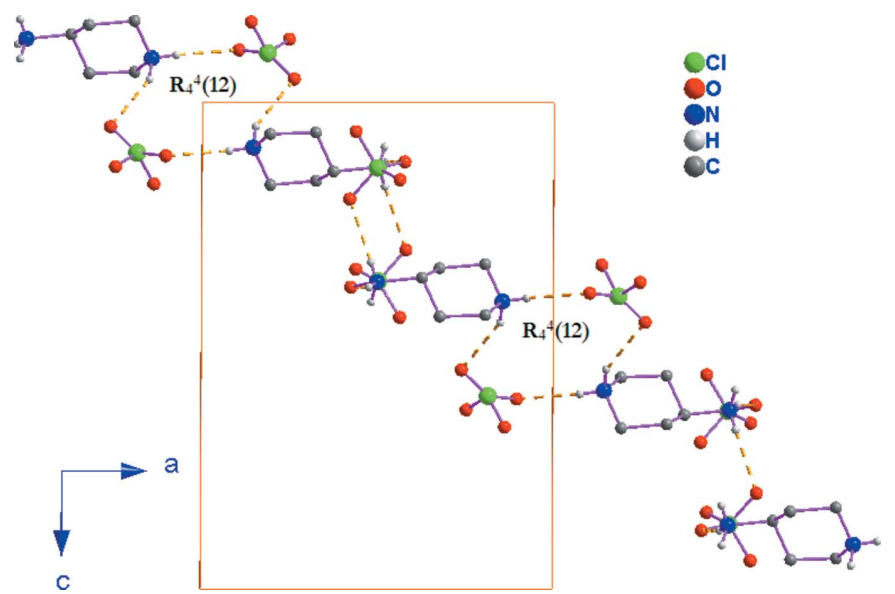

Figure 3

Chaîne ondulée se developant selon la direction [201] donnant lieu aux motifs de liaisons hydrogène $R_{4}^{4}(12)$.

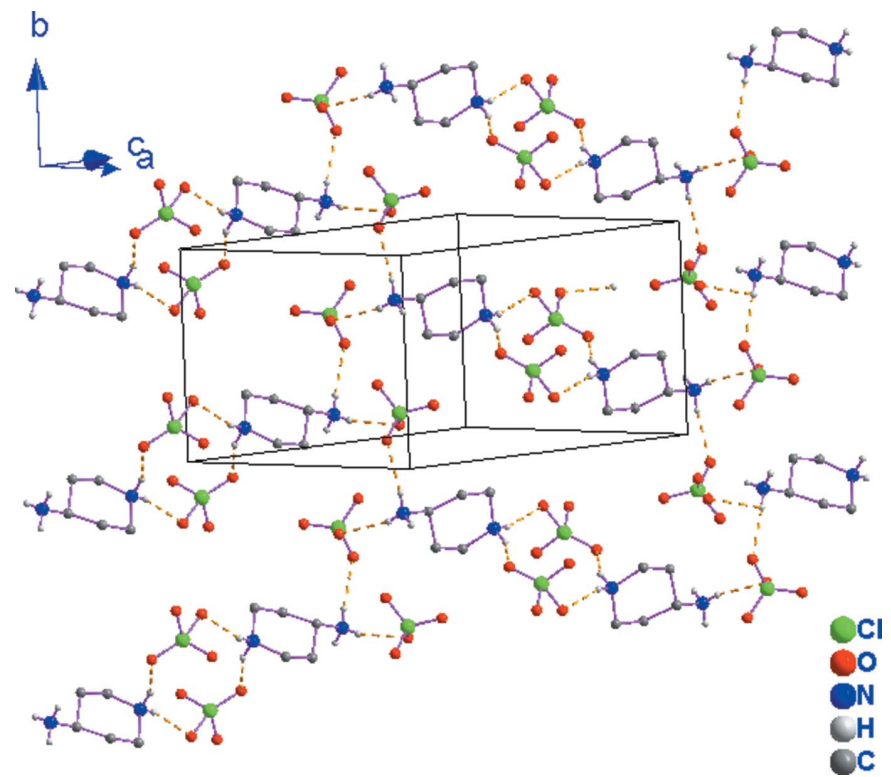

Figure 4

Chaînes ondulées formant une couche mixte dans la structure de (I) montrant les liaisons hydrogène de type $\mathrm{N}-\mathrm{H} \cdots \mathrm{O}$, intra- et interchaînes.

\section{Analyse thermique}

L'étude thermique est réalisée à l'aide d'un thermoanalyseur de type Setaram-Labsys Evo TG-DSC dans lequel un creuset vide est utilisé comme référence. L'analyse thermogravimétrique est effectuée avec $5,9 \mathrm{mg}$ de produit placé dans un creuset en platine. Le chauffage se fait de l'ambiante jusqu'à $723 \mathrm{~K}$ avec une vitesse de $5 \mathrm{~K} \mathrm{~min}^{-1}$ sous atmosphère d'argon.

Le thermogramme TG-DSC du composé $\left(\mathrm{C}_{9} \mathrm{H}_{22} \mathrm{~N}_{2}\right)\left[\mathrm{ClO}_{4}\right]_{2}$ est représenté sur la Fig. 5. La courbe TG révèle une seule perte de masse importante dans le domaine $543-578 \mathrm{~K}$, ce qui prouve que le composé étudié est dépourvu de molécules d'eau de cristallisation. En revanche la courbe DSC indique

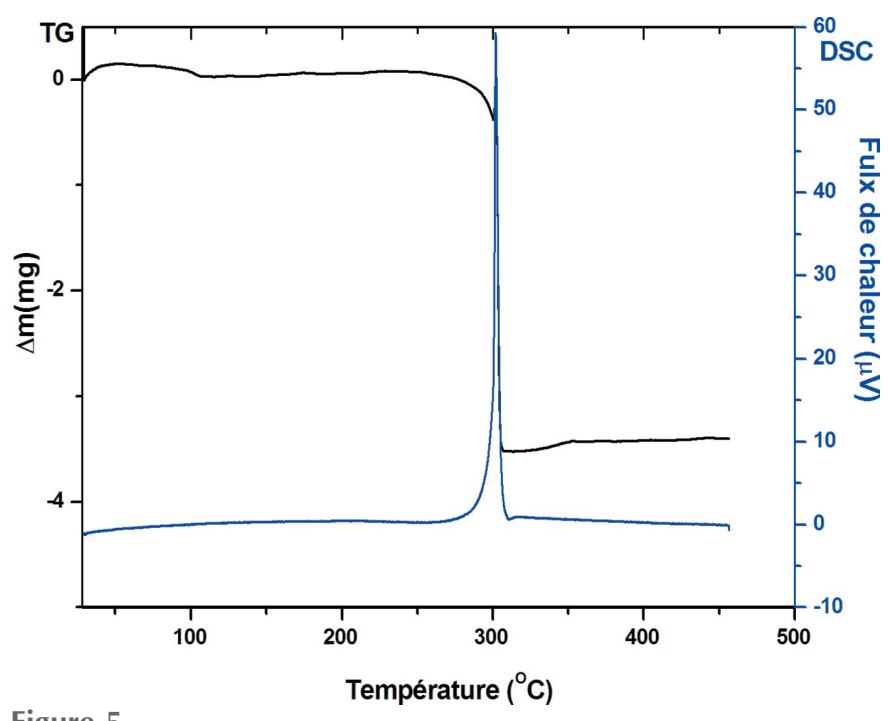

Figure 5

Courbes TG-DSC du composé (I). 


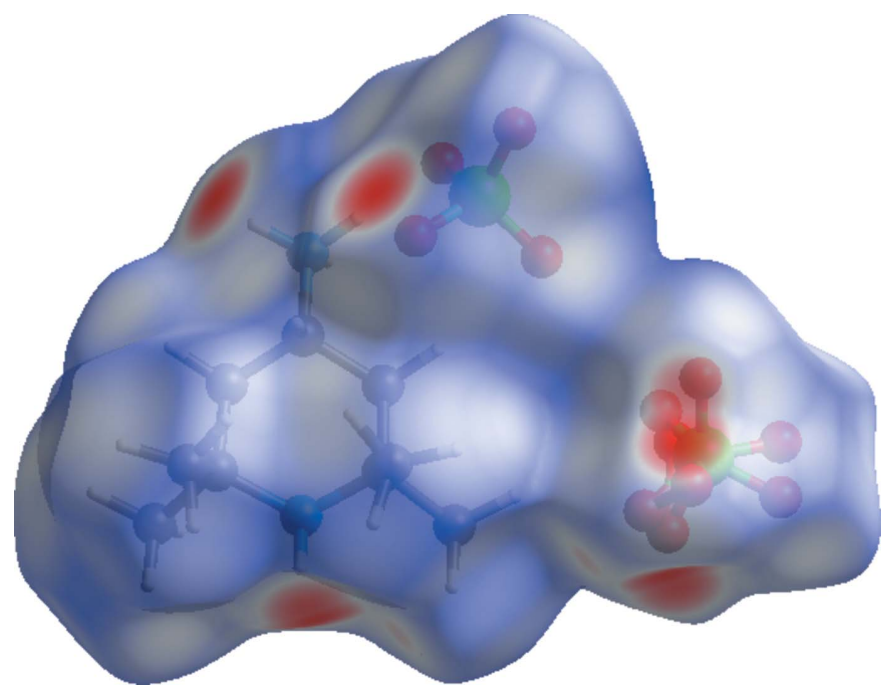

Figure 6

Surface Hirshfeld en mode $d_{\text {norm }}$ du composé (I).

un seul pic exothermique à $573 \mathrm{~K}$, qui correspond à la décomposition de la partie organique du composé (I).

\section{Analyse de surface Hirshfeld}

La représentation de la surface Hirshfeld du cation organique $\left(\mathrm{C}_{9} \mathrm{H}_{22} \mathrm{~N}_{2}\right)^{2+}$ et des deux anions $\mathrm{ClO}_{4}{ }^{-}$de l'unité asymétrique du composé (I) permet de mettre en évidence les liaisons hydrogène et les interactions intermoléculaires dans la structure cristalline (Spackman \& McKinnon, 2002; Spackman \& Jayatilaka, 2009). La surface Hirshfeld en modes $d_{\text {norm }}$ générée à l'aide du programme CrystalExplorer (Wolff et al., 2012) est illustrée dans la Fig. 6. Les tâches rouges correspondent aux contacts rapprochés $\mathrm{O} \cdots \mathrm{H} / \mathrm{H} \cdots \mathrm{O}$ qui sont dus aux liaisons hydrogène $\mathrm{N}-\mathrm{H} \cdots \mathrm{O}$. Les zones blanches marquent les endroits où la distance séparant les atomes voisins avoisine la somme des rayons de van der Waals des atomes considérés, elles indiquent des interactions de type $\mathrm{H} \cdots \mathrm{H}$. Les zones bleutées illustrent les domaines où les atomes voisins sont trop éloignés pour interagir entre eux.

Les empreintes digitales 2D de la surface Hirshfeld de la structure étudiée permettent de mettre en évidence les atomes participant à des contacts rapprochés (Parkin et al., 2007; Rohl et al., 2008). La Fig. 7 a illustre l'empreinte 2D de la totalité des contacts contribuant à la surface Hirshfeld. Le graphique exposé dans la Fig. $7 b$ représente les contacts $\mathrm{H} \cdots \mathrm{O} / \mathrm{O} \cdots \mathrm{H}$ entre les atomes d'hydrogène situés à l'intérieur de la surface Hirshfeld et les atomes d'oxygène situés à l'extérieur et réciproquement. Il est caractérisé par deux pointes symétriques situées en haut et à gauche et en bas à droite avec $d_{e}+d_{i}=2 \AA$ A Ces données sont caractéristiques des liaisons hydrogène $\mathrm{N}-$ H. . O. Elles ont la contribution la plus importante à la surface Hirshfeld totale $(73,3 \%)$. Le graphique représenté dans la Fig. $7 c$ illustre l'empreinte 2D des points $\left(d_{i}, d_{e}\right)$ associés aux atomes d'hydrogène $\left(\mathrm{r}_{\mathrm{vdW}}=1,20 \AA\right)$. Il est caractérisé par une extrémité qui pointe vers l'origine selon la diagonale et qui correspond à $d_{i} \sim d_{e} \sim 1,2 \AA$, ce qui révèle la présence des contacts rapprochés $\mathrm{H} \cdot \cdots \mathrm{H}$ au sein du composé étudié. Ces contacts $\mathrm{H} \cdots \mathrm{H}$ représentent $22,0 \%$ de la totalité de tous les contacts intermoléculaires. La décomposition de l'empreinte digitale 2D montre aussi d'autres contacts: $\mathrm{O} \cdots \mathrm{O}(4,6 \%$, Fig. $7 d)$ et $\mathrm{Cl} \cdots \mathrm{H} / \mathrm{H} \cdots \mathrm{Cl}(0,1 \%)$.

\section{Synthèse et cristallisation}

Le composé $\left(\mathrm{C}_{9} \mathrm{H}_{22} \mathrm{~N}_{2}\right)\left[\mathrm{ClO}_{4}\right]_{2}$ est obtenu en mélangeant dans l'eau, le 4-amino-2,2,6,6-tétraméthylpipéridine (pureté $\geq 97 \%$, Sigma-Aldrich) et l'acide perchlorique (pureté 70\%, Merck) selon les proportions molaire 1: 2. Après agitation, la solution finale est laissée évaporer à température ambiante. Après quelques jours, des cristaux incolores sous forme de parallélépipèdes commencent à apparaître. Ils ont une taille optimale pour une étude structurale.

\section{Affinement}

Les atomes d'hydrogène liés aux atomes de carbone ont été fixés dans leurs positions géométriques calculés en appliquant les contraintes suivantes: $\mathrm{C}-\mathrm{H}=0,96 \AA$ A pour le groupement $\mathrm{CH}_{3}$ avec $U_{\text {iso }}(\mathrm{H})=1,5 U_{\text {eq }}(\mathrm{C})$ et $\mathrm{C}-\mathrm{H}=0,97 \AA$ pour le groupement $-\mathrm{CH}_{2}$ avec $U_{\text {iso }}(\mathrm{H})=1,2 U_{\text {eq }}(\mathrm{C})$. Les données cristallographiques, les conditions de la collecte et les résultats de l'affinement de la structure du composé (I) sont regroupés dans le Tableau 2.

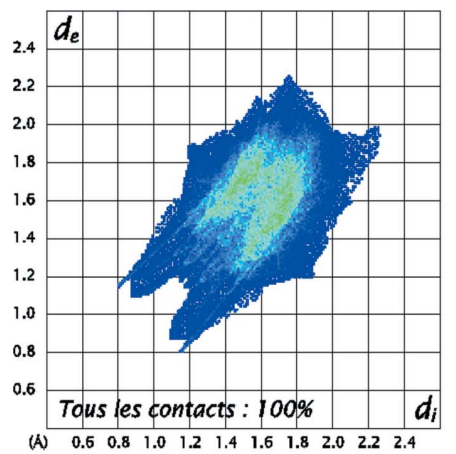

(a)

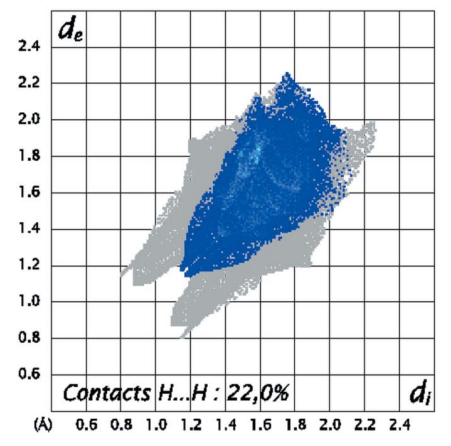

(c)

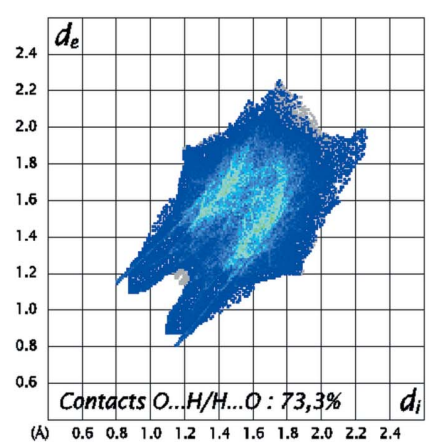

(b)

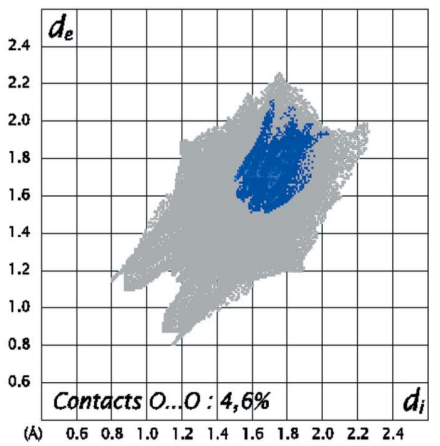

(d)
Figure 7

Empreintes digitales bidimensionnelles du composé (I): tous les contacts intermoléculaires $(a)$, contacts $\mathrm{O} \cdots \mathrm{H} / \mathrm{H} \cdots \mathrm{O}(b), \mathrm{H} \cdots \mathrm{H}(c)$ et $\mathrm{O} \cdots \mathrm{O}(d)$. 
Tableau 2

Détails expérimentaux.

\begin{tabular}{|c|c|}
\hline \multicolumn{2}{|l|}{ Données cristallines } \\
\hline Formule chimique & $\mathrm{C}_{9} \mathrm{H}_{22} \mathrm{~N}_{2}^{2+} \cdot 2 \mathrm{ClO}_{4}^{-}$ \\
\hline$M_{\mathrm{r}}$ & 357,18 \\
\hline Système cristallin, groupe d'espace & Monoclinique, $P 2_{1} / c$ \\
\hline Température $(\mathrm{K})$ & 293 \\
\hline$a, b, c(\AA)$ & $11,690(4), 8,330(2), 16,160(5)$ \\
\hline$\beta\left(^{\circ}\right)$ & $90,31(2)$ \\
\hline$V\left(\AA^{3}\right)$ & $1573,6(8)$ \\
\hline$Z$ & 4 \\
\hline Type de rayonnement & Мо $K \alpha$ \\
\hline$\mu\left(\mathrm{mm}^{-1}\right)$ & 0,45 \\
\hline Taille du cristal (mm) & $0,9 \times 0,54 \times 0,36$ \\
\hline \multicolumn{2}{|l|}{ Collecte de données } \\
\hline Diffractomètre & Enraf-Nonius CAD-4 \\
\hline Correction d'absorption & $\psi$ scan (North et al.,1968) \\
\hline$T_{\min }, T_{\max }$ & $0,87,0,99$ \\
\hline $\begin{array}{l}\text { Nombre de réflexions mesurées, } \\
\text { indépendantes et observées }[I> \\
2 \sigma(I)]\end{array}$ & $4106,3423,2193$ \\
\hline$R_{\text {int }}$ & 0,028 \\
\hline \multicolumn{2}{|l|}{ Affinement } \\
\hline$R\left[F^{2}>2 \sigma\left(F^{2}\right)\right], w R\left(F^{2}\right), S$ & $0,061,0,176,1,04$ \\
\hline Nombre de réflexions & 3077 \\
\hline Nombre de paramètres & 251 \\
\hline Nombre de restraints & 76 \\
\hline $\begin{array}{l}\text { Traitement des atomes d'hydro- } \\
\text { gène }\end{array}$ & $\begin{array}{l}\text { H atomes traitées par un mélange } \\
\text { d'affinement indépendant et } \\
\text { contraint }\end{array}$ \\
\hline$\Delta \rho_{\max }, \Delta \rho_{\min }\left(\mathrm{e} \AA^{-3}\right)$ & $0,50,-0,31$ \\
\hline
\end{tabular}

Programmes informatiques:: CAD-4 EXPRESS (Duisenberg, 1992; Macíček \& Yordanov, 1992), XCAD4 (Harms \& Wocadlo, 1995), SHELXS97 (Sheldrick, 2008), SHELXL2014/7 (Sheldrick, 2015), DIAMOND (Brandenburg, 2006), WinGX (Farrugia, 2012) et publCIF (Westrip, 2010).

\section{Remerciements}

Soutien financier du ministère de l'enseignement supérieur et recherche scientifique de la Tunisie tient à reconnaître. Nous remercions vivement Dr Ridha Ben Smail (Institut Préparatoire aux Etudes d'Ingénieurs de Nabeul) pour les discussions fructueuses.

\section{Références}

Anitha, R., Athimoolam, S., Gunasekaran, M. \& Sridhar, B. (2013). Acta Cryst. E69, o1236.

Avecilla, F., Esteban, D., Platas-Iglesias, C., Fernández-Martínez, S., De Blas, A. \& Rodríguez-Blas, T. (2005). Acta Cryst. C61, o92-o94.

Bendjeddou, L., Cherouana, A., Dahaoui, S., Benali-Cherif, N. \& Lecomte, C. (2003). Acta Cryst. E59, o649-o651.

Bouchouit, K., Essaidi, Z., Abed, S., Migalska-Zalas, A., Derkowska, B., Benali-cherif, N., Mihaly, M., Meghea, A. \& Sahraoui, B. (2008). Chem. Phys. Lett. 455, 270-274.
Brandenburg, K. (2006). DIAMOND. Crystal Impact GRb, Bonn, Allemagne.

Butcher, R. J., Gultneh, Y. \& Khan, A. R. (2002). Acta Cryst. E58, o1204-o1206.

Chebbi, H., Ben Smail, R. \& Zid, M. F. (2014). Acta Cryst. E70, 0642.

Chebbi, H. \& Driss, A. (2001). Acta Cryst. C57, 1369-1370.

Cui, Z., Gao, K., Liu, C., Yin, Y., Fu, D.-W., Cai, H.-L. \& Wu, X. S. (2016). J. Phys. Chem. C, 120, 2925-2931.

Czarnecki, P., Nawrocik, W., Pajak, Z. \& Wasicki, J. (1994). J. Phys. Condens. Matter, 6, 4955-4960.

Dai, J. (2008). Acta Cryst. E64, o2025.

Direm, A., Altomare, A., Moliterni, A. \& Benali-Cherif, N. (2015). Acta Cryst. B71, 427-436.

Drozd, M. \& Dudzic, D. (2013). Spectrochim. Acta A Mol. Biomol. Spectrosc. 115, 345-356.

Duisenberg, A. J. M. (1992). J. Appl. Cryst. 25, 92-96.

Farrugia, L. J. (2012). J. Appl. Cryst. 45, 849-854.

Gholizadeh, M., Pourayoubi, M., Farimaneh, M., Tarahhomi, A., Dušek, M. \& Eigner, V. (2014). Acta Cryst. C70, 230-235.

Harms, K. \& Wocadlo, S. (1995). XCAD4. Université de Marburg, Allemagne.

Huang, P.-M. \& Deng, Y. (2007). Acta Cryst. E63, o4170.

Jeffrey, G. A. (1997). An Introduction to Hydrogen Bonding. Oxford University Press.

Kaabi, K., El Glaoui, M., Ferretti, V., Zeller, M. \& Ben Nasr, C. (2011). Acta Cryst. E67, o2507-o2508.

Kaabi, K., El Glaoui, M., Jeanneau, E., Rzaigui, M. \& Ben Nasr, C. (2010). Acta Cryst. E66, o1722.

Macíček, J. \& Yordanov, A. (1992). J. Appl. Cryst. 25, 73-80.

Mackenzie, J. D. (1993). J. Sol-Gel Sci. Technol. 1, 7-19.

Mammeri, F., Bourhis, E. L., Rozes, L. \& Sanchez, C. (2005). J. Mater. Chem. 15, 3787-3811.

Marchewka, M. K. \& Drozd, M. (2013). Cent. Eur. J. Chem. 11(8), 1264-1277.

Mrad, M. L., Akriche, S., Rzaigui, M. \& Ben Nasr, C. (2009). Acta Cryst. E65, o757-0758.

North, A. C. T., Phillips, D. C. \& Mathews, F. S. (1968). Acta Cryst. A24, 351-359.

Parkin, A., Barr, G., Dong, W., Gilmore, C. J., Jayatilaka, D., McKinnon, J. J., Spackman, M. A. \& Wilson, C. C. (2007). CrystEngComm, 9, 648-652.

Rohl, A. L., Moret, M., Kaminsky, W., Claborn, K., McKinnon, J. J. \& Kahr, B. (2008). Cryst. Growth Des. 8, 4517-4525.

Sheldrick, G. M. (2008). Acta Cryst. A64, 112-122.

Sheldrick, G. M. (2015). Acta Cryst. C71, 3-8.

Spackman, M. A. \& Jayatilaka, D. (2009). CrystEngComm, 11, 19-32.

Spackman, M. A. \& McKinnon, J. J. (2002). CrystEngComm, 4, 378392.

Sutha Siluvai, G., Varghese, B. \& Murthy, N. N. (2005). Acta Cryst. C61, o380-o382.

Vivek, P., Suvitha, A. \& Murugakoothan, P. (2015). Spectrochim. Acta A Mol. Biomol. Spectrosc. 134, 517-525.

Westrip, S. P. (2010). J. Appl. Cryst. 43, 920-925.

Wolff, S. K., Grimwood, D. J., McKinnon, J. J., Turner, M. J., Jayatilaka, D. \& Spackman, M. A. (2012). Crystal Explorer. Université de Western Australia.

Zhang, L. (2009). Acta Cryst. E65, o2422.

Zhao, M.-M. (2012). Acta Cryst. E68, o327. 


\section{supporting information}

Acta Cryst. (2017). E73, 1453-1457 [https://doi.org/10.1107/S2056989017012695]

Structure cristalline et analyses thermique et de surface Hirshfeld du diperchlorate de 4-azaniumyl-2,2,6,6-tétraméthylpipéridin-1-ium

\section{Hammouda Chebbi, Abdessalem Boumakhla, Mohamed Faouzi Zid et Abderrahmen Guesmi}

Computing details

Data collection: CAD-4 EXPRESS (Duisenberg, 1992; Macíček \& Yordanov, 1992); cell refinement: CAD-4 EXPRESS

(Duisenberg, 1992; Macíček \& Yordanov, 1992); data reduction: XCAD4 (Harms \& Wocadlo, 1995); program(s) used to solve structure: SHELXS97 (Sheldrick, 2008); program(s) used to refine structure: SHELXL2014/7 (Sheldrick, 2015); molecular graphics: DIAMOND (Brandenburg, 2006); software used to prepare material for publication: WinGX (Farrugia, 2012) et publCIF (Westrip, 2010).

4-Azaniumyl-2,2,6,6-tétraméthylpipéridin-1-ium diperchlorate

Crystal data

$\mathrm{C}_{9} \mathrm{H}_{22} \mathrm{~N}_{2}{ }^{2+} \cdot 2 \mathrm{ClO}_{4}^{-}$

$M_{r}=357.18$

Monoclinic, $P 2_{1} / c$

$a=11.690(4) \AA$

$b=8.330(2) \AA$

$c=16.160(5) \AA$

$\beta=90.31(2)^{\circ}$

$V=1573.6(8) \AA^{3}$

$Z=4$

Data collection

Enraf-Nonius CAD-4 diffractometer

Radiation source: fine-focus sealed tube

Graphite monochromator

w $/ 2 \theta$ scans

Absorption correction: $\psi$ scan

(North et al.,1968)

$T_{\min }=0.87, T_{\max }=0.99$

4106 measured reflections

Refinement

Refinement on $F^{2}$

Least-squares matrix: full

$R\left[F^{2}>2 \sigma\left(F^{2}\right)\right]=0.061$

$w R\left(F^{2}\right)=0.176$

$S=1.04$

3077 reflections
$F(000)=752$

$D_{\mathrm{x}}=1.508 \mathrm{Mg} \mathrm{m}^{-3}$

Mo $K \alpha$ radiation, $\lambda=0.71073 \AA$

Cell parameters from 25 reflections

$\theta=12-15^{\circ}$

$\mu=0.45 \mathrm{~mm}^{-1}$

$T=293 \mathrm{~K}$

Parallelepiped, colorless

$0.9 \times 0.54 \times 0.36 \mathrm{~mm}$

3423 independent reflections

2193 reflections with $I>2 \sigma(I)$

$R_{\text {int }}=0.028$

$\theta_{\max }=27.0^{\circ}, \theta_{\min }=2.5^{\circ}$

$h=-14 \rightarrow 1$

$k=-1 \rightarrow 10$

$l=-20 \rightarrow 20$

2 standard reflections every 120 reflections intensity decay: $1 \%$

251 parameters

76 restraints

Hydrogen site location: mixed

$\mathrm{H}$ atoms treated by a mixture of independent and constrained refinement 
$w=1 /\left[\sigma^{2}\left(F_{\mathrm{o}}^{2}\right)+(0.0865 P)^{2}+1.3556 P\right]$

where $P=\left(F_{\mathrm{o}}^{2}+2 F_{\mathrm{c}}^{2}\right) / 3$

$(\Delta / \sigma)_{\max }<0.001$

$$
\Delta \rho_{\max }=0.50 \mathrm{e} \AA^{-3}
$$

\section{Special details}

Geometry. All esds (except the esd in the dihedral angle between two 1.s. planes) are estimated using the full covariance matrix. The cell esds are taken into account individually in the estimation of esds in distances, angles and torsion angles; correlations between esds in cell parameters are only used when they are defined by crystal symmetry. An approximate (isotropic) treatment of cell esds is used for estimating esds involving 1.s. planes.

Fractional atomic coordinates and isotropic or equivalent isotropic displacement parameters $\left(\AA^{2}\right)$

\begin{tabular}{|c|c|c|c|c|c|}
\hline & $x$ & $y$ & $z$ & $U_{\text {iso }} * / U_{\text {eq }}$ & Occ. $(<1)$ \\
\hline $\mathrm{C} 11$ & $0.50151(7)$ & 0.31499 (12) & $-0.13350(6)$ & 0.0489 & \\
\hline $\mathrm{Cl} 2$ & $0.81520(6)$ & $0.14325(11)$ & $0.10249(6)$ & $0.0451(3)$ & \\
\hline O1 & $0.4382(3)$ & $0.1784(4)$ & -0.1580 & $0.0826(10)$ & \\
\hline $\mathrm{O} 2$ & $0.4241(2)$ & $0.4462(4)$ & $-0.1214(2)$ & $0.0737(9)$ & \\
\hline $\mathrm{O} 3$ & $0.5804(2)$ & $0.3560(5)$ & $-0.1981(2)$ & $0.0792(10)$ & \\
\hline $\mathrm{O} 4$ & 0.5640 & $0.2872(5)$ & $-0.0592(2)$ & $0.0813(10)$ & \\
\hline $\mathrm{O} 5 \mathrm{~A}$ & $0.7387(5)$ & $0.0548(7)$ & $0.0500(4)$ & $0.086(2)$ & $0.625(7)$ \\
\hline O6A & $0.7578(9)$ & $0.2766(13)$ & $0.1287(8)$ & $0.123(4)$ & $0.625(7)$ \\
\hline O7A & $0.8971(10)$ & $0.2623(16)$ & $0.1176(14)$ & $0.194(6)$ & $0.625(7)$ \\
\hline O8A & $0.8629(8)$ & $0.0545(13)$ & $0.1667(5)$ & $0.115(3)$ & $0.625(7)$ \\
\hline O5B & $0.777(2)$ & $0.071(3)$ & $0.1711(9)$ & $0.164(8)$ & $0.375(7)$ \\
\hline O6B & $0.7303(15)$ & $0.252(3)$ & $0.096(2)$ & $0.221(14)$ & $0.375(7)$ \\
\hline O7B & $0.9060(7)$ & $0.196(2)$ & $0.0523(8)$ & $0.090(4)$ & $0.375(7)$ \\
\hline O8B & $0.8933(9)$ & $0.068(2)$ & $0.0508(10)$ & $0.124(6)$ & $0.375(7)$ \\
\hline N1 & $0.8554(2)$ & $0.8496(3)$ & $-0.09235(18)$ & $0.0353(6)$ & \\
\hline $\mathrm{H} 1 \mathrm{~A}$ & $0.931(3)$ & $0.831(4)$ & $-0.100(2)$ & $0.028(8)^{*}$ & \\
\hline H1B & $0.843(3)$ & $0.914(5)$ & $-0.053(3)$ & $0.042(10)^{*}$ & \\
\hline N2 & $0.4985(3)$ & $0.7836(6)$ & $-0.1322(3)$ & $0.0631(10)$ & \\
\hline $\mathrm{H} 2 \mathrm{~A}$ & $0.469(5)$ & $0.689(10)$ & $-0.124(5)$ & $0.11(2)^{*}$ & \\
\hline $\mathrm{H} 2 \mathrm{~B}$ & $0.476(4)$ & $0.818(7)$ & $-0.184(4)$ & $0.077(15)^{*}$ & \\
\hline $\mathrm{H} 2 \mathrm{C}$ & $0.480(4)$ & $0.831(7)$ & $-0.086(2)$ & $0.086(19)^{*}$ & \\
\hline $\mathrm{C} 1$ & $0.8071(3)$ & 0.6875 & $-0.0636(2)$ & $0.0382(7)$ & \\
\hline $\mathrm{C} 2$ & 0.6774 & $0.7041(4)$ & $-0.0605(2)$ & $0.0430(8)$ & \\
\hline $\mathrm{H} 2 \mathrm{D}$ & 0.6572 & 0.7757 & -0.0155 & $0.052 *$ & \\
\hline $\mathrm{H} 2 \mathrm{E}$ & 0.6441 & 0.5999 & -0.0488 & $0.052 *$ & \\
\hline $\mathrm{C} 3$ & $0.6264(2)$ & $0.7688(4)$ & $-0.1410(2)$ & $0.0410(7)$ & \\
\hline H3 & 0.6439 & 0.6948 & -0.1863 & $0.049 *$ & \\
\hline $\mathrm{C} 4$ & 0.6758 & $0.9335(4)$ & $-0.1603(2)$ & $0.0404(7)$ & \\
\hline $\mathrm{H} 4 \mathrm{~A}$ & 0.6543 & 1.0074 & -0.1166 & $0.048 *$ & \\
\hline H4B & 0.6422 & 0.9723 & -0.2115 & $0.048^{*}$ & \\
\hline $\mathrm{C} 5$ & 0.8058 & $0.9342(4)$ & -0.1685 (2) & $0.0365(7)$ & \\
\hline C6 & $0.8472(3)$ & $0.8544(6)$ & $-0.2482(2)$ & $0.0592(10)$ & \\
\hline H6A & 0.8104 & 0.7521 & -0.2546 & $0.089^{*}$ & \\
\hline H6B & 0.9286 & 0.8396 & -0.2453 & $0.089^{*}$ & \\
\hline $\mathrm{H} 6 \mathrm{C}$ & 0.8286 & 0.9215 & -0.2946 & $0.089 *$ & \\
\hline $\mathrm{C} 7$ & $0.8506(3)$ & $1.1078(5)$ & -0.1640 & $0.0579(10)$ & \\
\hline
\end{tabular}




\begin{tabular}{lllll} 
H7A & 0.8181 & 1.1695 & -0.2084 & $0.087^{*}$ \\
H7B & 0.9324 & 1.1076 & -0.1686 & $0.087^{*}$ \\
H7C & 0.8291 & 1.1546 & -0.1120 & $0.087^{*}$ \\
C8 & $0.8546(3)$ & $0.6613(5)$ & $0.0239(3)$ & $0.0566(10)$ \\
H8A & 0.8321 & 0.7491 & 0.0587 & $0.085^{*}$ \\
H8B & 0.9365 & 0.6555 & 0.0220 & $0.085^{*}$ \\
H8C & 0.8248 & 0.5628 & 0.0459 & $0.085^{*}$ \\
C9 & $0.8465(4)$ & $0.5516(5)$ & $-0.1205(3)$ & $0.0624(11)$ \\
H9A & 0.8348 & 0.4503 & -0.0935 & $0.094^{*}$ \\
H9B & 0.9262 & 0.5647 & -0.1326 & $0.094^{*}$ \\
H9C & 0.8030 & 0.5545 & -0.1711 & $0.094^{*}$ \\
\hline
\end{tabular}

Atomic displacement parameters $\left(\AA^{2}\right)$

\begin{tabular}{|c|c|c|c|c|c|c|}
\hline & $U^{11}$ & $U^{22}$ & $U^{33}$ & $U^{12}$ & $U^{13}$ & $U^{23}$ \\
\hline $\mathrm{Cl1}$ & $0.0460(5)$ & $0.0553(6)$ & $0.0455(5)$ & $0.0046(4)$ & $-0.0021(3)$ & $-0.0024(4)$ \\
\hline $\mathrm{Cl} 2$ & 0.0418 (4) & $0.0486(5)$ & $0.0449(5)$ & $0.0028(3)$ & $0.0007(3)$ & $-0.0056(4)$ \\
\hline $\mathrm{O} 1$ & $0.077(2)$ & $0.067(2)$ & $0.103(3)$ & $-0.0131(16)$ & $-0.0159(18)$ & $-0.0022(19)$ \\
\hline $\mathrm{O} 2$ & $0.0682(18)$ & $0.067(2)$ & $0.086(2)$ & $0.0199(15)$ & 0.0057 (15) & 0.0007 (17) \\
\hline $\mathrm{O} 3$ & $0.0642(17)$ & $0.109(3)$ & $0.065(2)$ & $-0.0054(17)$ & $0.0111(14)$ & -0.0009 (19) \\
\hline $\mathrm{O} 4$ & $0.080(2)$ & 0.107 (3) & 0.0565 (19) & 0.0245 (19) & $-0.0170(15)$ & $-0.0021(19)$ \\
\hline O5A & $0.104(4)$ & $0.066(4)$ & $0.086(4)$ & -0.010 & $-0.042(3)$ & $-0.023(3)$ \\
\hline O6A & $0.112(8)$ & $0.102(6)$ & $0.154(8)$ & $0.045(5)$ & $-0.056(6)$ & $-0.076(6)$ \\
\hline O7A & $0.157(9)$ & $0.134(9)$ & $0.290(18)$ & $-0.052(7)$ & $0.034(10)$ & $-0.035(11)$ \\
\hline O8A & $0.139(6)$ & $0.130(7)$ & $0.075(5)$ & $0.038(6)$ & $-0.045(5)$ & $0.024(5)$ \\
\hline O5B & 0.237 (19) & $0.18(2)$ & $0.072(10)$ & $-0.076(18)$ & $0.042(13)$ & $0.037(10)$ \\
\hline O6B & $0.050(8)$ & $0.27(3)$ & $0.35(4)$ & $0.043(12)$ & $-0.022(14)$ & $0.05(3)$ \\
\hline O7B & $0.038(4)$ & $0.154(12)$ & $0.077(7)$ & $-0.013(6)$ & $0.016(4)$ & $0.019(8)$ \\
\hline O8B & $0.082(7)$ & $0.149(12)$ & $0.142(12)$ & $-0.017(8)$ & $0.040(7)$ & $-0.092(10)$ \\
\hline N1 & $0.0382(14)$ & $0.0327(14)$ & $0.0351(15)$ & $-0.0004(10)$ & $-0.0071(10)$ & $0.0018(12)$ \\
\hline N2 & $0.0418(17)$ & $0.081(3)$ & $0.067(3)$ & $-0.0056(17)$ & $-0.0101(16)$ & $0.013(2)$ \\
\hline $\mathrm{C} 1$ & $0.0445(16)$ & $0.0292(15)$ & 0.0407 (18) & $-0.0008(12)$ & $-0.0071(13)$ & $0.0073(13)$ \\
\hline $\mathrm{C} 2$ & 0.0485 (17) & $0.0421(18)$ & 0.0383 (19) & $-0.0083(14)$ & $-0.0020(13)$ & $0.0079(14)$ \\
\hline $\mathrm{C} 3$ & $0.0361(15)$ & 0.0457 (19) & $0.0410(18)$ & $-0.0025(13)$ & $-0.0067(12)$ & $0.0015(15)$ \\
\hline $\mathrm{C} 4$ & $0.0456(17)$ & 0.0357 (16) & 0.0397 (18) & 0.0034 (13) & $-0.0084(12)$ & $0.0063(14)$ \\
\hline $\mathrm{C} 5$ & $0.0444(16)$ & $0.0342(16)$ & $0.0306(16)$ & $-0.0041(12)$ & $-0.0039(11)$ & $0.0054(13)$ \\
\hline C6 & $0.073(2)$ & $0.069(3)$ & 0.0355 (19) & $0.000(2)$ & 0.0089 (16) & 0.0007 (18) \\
\hline C7 & $0.074(2)$ & $0.040(2)$ & $0.059(2)$ & $-0.0170(18)$ & $-0.0132(18)$ & 0.0145 (18) \\
\hline $\mathrm{C} 8$ & $0.070(2)$ & $0.052(2)$ & $0.047(2)$ & $-0.0027(17)$ & $-0.0189(17)$ & 0.0178 (18) \\
\hline C9 & $0.079(3)$ & $0.037(2)$ & $0.070(3)$ & 0.0173 (18) & $-0.008(2)$ & -0.0087 (19) \\
\hline
\end{tabular}

Geometric parameters $\left(\AA,{ }^{\circ}\right)$

\begin{tabular}{llll}
\hline $\mathrm{C} 11-\mathrm{O} 1$ & $1.413(3)$ & $\mathrm{C} 1-\mathrm{C} 8$ & $1.532(5)$ \\
$\mathrm{Cl1}-\mathrm{O} 4$ & $1.422(3)$ & $\mathrm{C} 2-\mathrm{C} 3$ & $1.526(5)$ \\
$\mathrm{Cl1}-\mathrm{O} 2$ & $1.433(3)$ & $\mathrm{C} 2-\mathrm{H} 2 \mathrm{D}$ & 0.9700 \\
$\mathrm{Cl1}-\mathrm{O} 3$ & $1.438(3)$ & $\mathrm{C} 2-\mathrm{H} 2 \mathrm{E}$ & 0.9700 \\
$\mathrm{Cl} 2-\mathrm{O} 5 \mathrm{~B}$ & $1.338(11)$ & $\mathrm{C} 3-\mathrm{C} 4$ & $1.522(5)$
\end{tabular}




\begin{tabular}{|c|c|c|c|}
\hline $\mathrm{Cl} 2-\mathrm{O} 6 \mathrm{~B}$ & 1.349 (14) & $\mathrm{C} 3-\mathrm{H} 3$ & 0.9800 \\
\hline $\mathrm{Cl} 2-\mathrm{O} 6 \mathrm{~A}$ & $1.366(7)$ & $\mathrm{C} 4-\mathrm{C} 5$ & $1.526(4)$ \\
\hline $\mathrm{Cl} 2-\mathrm{O} 8 \mathrm{~A}$ & $1.388(7)$ & $\mathrm{C} 4-\mathrm{H} 4 \mathrm{~A}$ & 0.9700 \\
\hline $\mathrm{Cl} 2-\mathrm{O} 8 \mathrm{~B}$ & $1.390(9)$ & $\mathrm{C} 4-\mathrm{H} 4 \mathrm{~B}$ & 0.9700 \\
\hline $\mathrm{Cl} 2-\mathrm{O} 7 \mathrm{~A}$ & $1.399(9)$ & $\mathrm{C} 5-\mathrm{C} 6$ & $1.530(5)$ \\
\hline $\mathrm{Cl} 2-\mathrm{O} 7 \mathrm{~B}$ & $1.411(8)$ & $\mathrm{C} 5-\mathrm{C} 7$ & $1.539(5)$ \\
\hline $\mathrm{Cl} 2-\mathrm{O} 5 \mathrm{~A}$ & $1.434(5)$ & C6-H6A & 0.9600 \\
\hline $\mathrm{O} 6 \mathrm{~A}-\mathrm{O} 7 \mathrm{~A}$ & $1.644(16)$ & C6-H6B & 0.9600 \\
\hline $\mathrm{O} 7 \mathrm{~B}-\mathrm{O} 8 \mathrm{~B}$ & $1.08(2)$ & C6- $\mathrm{H} 6 \mathrm{C}$ & 0.9600 \\
\hline $\mathrm{N} 1-\mathrm{C} 5$ & $1.530(4)$ & C7-H7A & 0.9600 \\
\hline $\mathrm{N} 1-\mathrm{C} 1$ & $1.537(4)$ & C7-H7B & 0.9600 \\
\hline $\mathrm{N} 1-\mathrm{H} 1 \mathrm{~A}$ & $0.91(3)$ & $\mathrm{C} 7-\mathrm{H} 7 \mathrm{C}$ & 0.9600 \\
\hline $\mathrm{N} 1-\mathrm{H} 1 \mathrm{~B}$ & $0.84(4)$ & $\mathrm{C} 8-\mathrm{H} 8 \mathrm{~A}$ & 0.9600 \\
\hline $\mathrm{N} 2-\mathrm{C} 3$ & $1.508(4)$ & $\mathrm{C} 8-\mathrm{H} 8 \mathrm{~B}$ & 0.9600 \\
\hline $\mathrm{N} 2-\mathrm{H} 2 \mathrm{~A}$ & $0.87(8)$ & $\mathrm{C} 8-\mathrm{H} 8 \mathrm{C}$ & 0.9600 \\
\hline $\mathrm{N} 2-\mathrm{H} 2 \mathrm{~B}$ & $0.92(6)$ & $\mathrm{C} 9-\mathrm{H} 9 \mathrm{~A}$ & 0.9600 \\
\hline $\mathrm{N} 2-\mathrm{H} 2 \mathrm{C}$ & $0.870(10)$ & C9-H9B & 0.9600 \\
\hline $\mathrm{C} 1-\mathrm{C} 2$ & $1.524(4)$ & $\mathrm{C} 9-\mathrm{H} 9 \mathrm{C}$ & 0.9600 \\
\hline $\mathrm{C} 1-\mathrm{C} 9$ & $1.531(5)$ & & \\
\hline $\mathrm{O} 1-\mathrm{Cl1}-\mathrm{O} 4$ & $111.8(2)$ & $\mathrm{C} 3-\mathrm{C} 2-\mathrm{H} 2 \mathrm{E}$ & 109.0 \\
\hline $\mathrm{O} 1-\mathrm{Cl1}-\mathrm{O} 2$ & $108.8(2)$ & $\mathrm{H} 2 \mathrm{D}-\mathrm{C} 2-\mathrm{H} 2 \mathrm{E}$ & 107.8 \\
\hline $\mathrm{O} 4-\mathrm{Cl} 1-\mathrm{O} 2$ & $109.3(2)$ & $\mathrm{N} 2-\mathrm{C} 3-\mathrm{C} 4$ & $108.9(3)$ \\
\hline $\mathrm{O} 1-\mathrm{Cl1}-\mathrm{O} 3$ & $108.9(2)$ & $\mathrm{N} 2-\mathrm{C} 3-\mathrm{C} 2$ & $109.4(3)$ \\
\hline $\mathrm{O} 4-\mathrm{Cl1}-\mathrm{O} 3$ & $108.9(2)$ & $\mathrm{C} 4-\mathrm{C} 3-\mathrm{C} 2$ & $110.3(3)$ \\
\hline $\mathrm{O} 2-\mathrm{Cl1}-\mathrm{O} 3$ & $109.0(2)$ & $\mathrm{N} 2-\mathrm{C} 3-\mathrm{H} 3$ & 109.4 \\
\hline $\mathrm{O} 5 \mathrm{~B}-\mathrm{Cl} 2-\mathrm{O} 6 \mathrm{~B}$ & 96.8 (19) & $\mathrm{C} 4-\mathrm{C} 3-\mathrm{H} 3$ & 109.4 \\
\hline $\mathrm{O} 6 \mathrm{~A}-\mathrm{Cl} 2-\mathrm{O} 8 \mathrm{~A}$ & $113.4(7)$ & $\mathrm{C} 2-\mathrm{C} 3-\mathrm{H} 3$ & 109.4 \\
\hline $\mathrm{O} 5 \mathrm{~B}-\mathrm{Cl} 2-\mathrm{O} 8 \mathrm{~B}$ & $121.2(15)$ & $\mathrm{C} 3-\mathrm{C} 4-\mathrm{C} 5$ & $113.6(2)$ \\
\hline $\mathrm{O} 6 \mathrm{~B}-\mathrm{Cl} 2-\mathrm{O} 8 \mathrm{~B}$ & $138.0(18)$ & $\mathrm{C} 3-\mathrm{C} 4-\mathrm{H} 4 \mathrm{~A}$ & 108.8 \\
\hline $\mathrm{O} 6 \mathrm{~A}-\mathrm{Cl} 2-\mathrm{O} 7 \mathrm{~A}$ & $72.9(8)$ & $\mathrm{C} 5-\mathrm{C} 4-\mathrm{H} 4 \mathrm{~A}$ & 108.8 \\
\hline $\mathrm{O} 8 \mathrm{~A}-\mathrm{Cl} 2-\mathrm{O} 7 \mathrm{~A}$ & $88.6(9)$ & $\mathrm{C} 3-\mathrm{C} 4-\mathrm{H} 4 \mathrm{~B}$ & 108.8 \\
\hline $\mathrm{O} 5 \mathrm{~B}-\mathrm{Cl} 2-\mathrm{O} 7 \mathrm{~B}$ & $150.4(12)$ & $\mathrm{C} 5-\mathrm{C} 4-\mathrm{H} 4 \mathrm{~B}$ & 108.8 \\
\hline $\mathrm{O} 6 \mathrm{~B}-\mathrm{Cl} 2-\mathrm{O} 7 \mathrm{~B}$ & $107.5(16)$ & $\mathrm{H} 4 \mathrm{~A}-\mathrm{C} 4-\mathrm{H} 4 \mathrm{~B}$ & 107.7 \\
\hline $\mathrm{O} 8 \mathrm{~B}-\mathrm{Cl} 2-\mathrm{O} 7 \mathrm{~B}$ & $45.2(8)$ & $\mathrm{C} 4-\mathrm{C} 5-\mathrm{C} 6$ & $113.1(3)$ \\
\hline $\mathrm{O} 6 \mathrm{~A}-\mathrm{Cl} 2-\mathrm{O} 5 \mathrm{~A}$ & $107.2(4)$ & $\mathrm{C} 4-\mathrm{C} 5-\mathrm{N} 1$ & $107.5(2)$ \\
\hline $\mathrm{O} 8 \mathrm{~A}-\mathrm{Cl} 2-\mathrm{O} 5 \mathrm{~A}$ & $114.5(5)$ & $\mathrm{C} 6-\mathrm{C} 5-\mathrm{N} 1$ & $110.9(3)$ \\
\hline $\mathrm{O} 7 \mathrm{~A}-\mathrm{Cl} 2-\mathrm{O} 5 \mathrm{~A}$ & $153.1(10)$ & $\mathrm{C} 4-\mathrm{C} 5-\mathrm{C} 7$ & $109.7(3)$ \\
\hline $\mathrm{Cl} 2-\mathrm{O} 6 \mathrm{~A}-\mathrm{O} 7 \mathrm{~A}$ & $54.5(6)$ & $\mathrm{C} 6-\mathrm{C} 5-\mathrm{C} 7$ & $109.9(3)$ \\
\hline $\mathrm{Cl} 2-\mathrm{O} 7 \mathrm{~A}-\mathrm{O} 6 \mathrm{~A}$ & $52.6(5)$ & $\mathrm{N} 1-\mathrm{C} 5-\mathrm{C} 7$ & $105.5(3)$ \\
\hline $\mathrm{O} 8 \mathrm{~B}-\mathrm{O} 7 \mathrm{~B}-\mathrm{Cl} 2$ & $66.4(7)$ & $\mathrm{C} 5-\mathrm{C} 6-\mathrm{H} 6 \mathrm{~A}$ & 109.5 \\
\hline $\mathrm{O} 7 \mathrm{~B}-\mathrm{O} 8 \mathrm{~B}-\mathrm{Cl} 2$ & $68.4(7)$ & $\mathrm{C} 5-\mathrm{C} 6-\mathrm{H} 6 \mathrm{~B}$ & 109.5 \\
\hline $\mathrm{C} 5-\mathrm{N} 1-\mathrm{C} 1$ & $120.7(2)$ & $\mathrm{H} 6 \mathrm{~A}-\mathrm{C} 6-\mathrm{H} 6 \mathrm{~B}$ & 109.5 \\
\hline $\mathrm{C} 5-\mathrm{N} 1-\mathrm{H} 1 \mathrm{~A}$ & $110(2)$ & $\mathrm{C} 5-\mathrm{C} 6-\mathrm{H} 6 \mathrm{C}$ & 109.5 \\
\hline $\mathrm{C} 1-\mathrm{N} 1-\mathrm{H} 1 \mathrm{~A}$ & $104(2)$ & $\mathrm{H} 6 \mathrm{~A}-\mathrm{C} 6-\mathrm{H} 6 \mathrm{C}$ & 109.5 \\
\hline $\mathrm{C} 5-\mathrm{N} 1-\mathrm{H} 1 \mathrm{~B}$ & $104(3)$ & $\mathrm{H} 6 \mathrm{~B}-\mathrm{C} 6-\mathrm{H} 6 \mathrm{C}$ & 109.5 \\
\hline $\mathrm{C} 1-\mathrm{N} 1-\mathrm{H} 1 \mathrm{~B}$ & $106(3)$ & $\mathrm{C} 5-\mathrm{C} 7-\mathrm{H} 7 \mathrm{~A}$ & 109.5 \\
\hline $\mathrm{H} 1 \mathrm{~A}-\mathrm{N} 1-\mathrm{H} 1 \mathrm{~B}$ & $112(3)$ & $\mathrm{C} 5-\mathrm{C} 7-\mathrm{H} 7 \mathrm{~B}$ & 109.5 \\
\hline
\end{tabular}




$\begin{array}{ll}\mathrm{C} 3-\mathrm{N} 2-\mathrm{H} 2 \mathrm{~A} & 110(4) \\ \mathrm{C} 3-\mathrm{N} 2-\mathrm{H} 2 \mathrm{~B} & 103(3) \\ \mathrm{H} 2 \mathrm{~A}-\mathrm{N} 2-\mathrm{H} 2 \mathrm{~B} & 108(6) \\ \mathrm{C} 3-\mathrm{N} 2-\mathrm{H} 2 \mathrm{C} & 112(3) \\ \mathrm{H} 2 \mathrm{~A}-\mathrm{N} 2-\mathrm{H} 2 \mathrm{C} & 100(6) \\ \mathrm{H} 2 \mathrm{~B}-\mathrm{N} 2-\mathrm{H} 2 \mathrm{C} & 124(5) \\ \mathrm{C} 2-\mathrm{C} 1-\mathrm{C} 9 & 112.9(3) \\ \mathrm{C} 2-\mathrm{C} 1-\mathrm{C} 8 & 109.7(3) \\ \mathrm{C} 9-\mathrm{C} 1-\mathrm{C} 8 & 109.9(3) \\ \mathrm{C} 2-\mathrm{C} 1-\mathrm{N} 1 & 107.3(2) \\ \mathrm{C} 9-\mathrm{C} 1-\mathrm{N} 1 & 110.9(3) \\ \mathrm{C} 8-\mathrm{C} 1-\mathrm{N} 1 & 105.9(3) \\ \mathrm{C} 1-\mathrm{C} 2-\mathrm{C} 3 & 112.8(3) \\ \mathrm{C} 1-\mathrm{C} 2-\mathrm{H} 2 \mathrm{D} & 109.0 \\ \mathrm{C} 3-\mathrm{C} 2-\mathrm{H} 2 \mathrm{D} & 109.0 \\ \mathrm{C} 1-\mathrm{C} 2-\mathrm{H} 2 \mathrm{E} & 109.0\end{array}$

$\begin{array}{ll}\text { H7A-C7-H7B } & 109.5 \\ \text { C5-C7-H7C } & 109.5 \\ \text { H7A-C7-H7C } & 109.5 \\ \text { H7B-C7-H7C } & 109.5 \\ \text { C1-C8-H8A } & 109.5 \\ \text { C1-C8-H8B } & 109.5 \\ \text { H8A-C8-H8B } & 109.5 \\ \text { C1-C8-H8C } & 109.5 \\ \text { H8A-C8-H8C } & 109.5 \\ \text { H8B-C8-H8C } & 109.5 \\ \text { C1-C9-H9A } & 109.5 \\ \text { C1-C9-H9B } & 109.5 \\ \text { H9A-C9-H9B } & 109.5 \\ \text { C1-C9-H9C } & 109.5 \\ \text { H9A-C9-H9C } & 109.5 \\ \text { H9B-C9-H9C } & 109.5\end{array}$

Hydrogen-bond geometry $\left(\AA,{ }^{\circ}\right)$

\begin{tabular}{lllll}
\hline$D-\mathrm{H} \cdots A$ & $D-\mathrm{H}$ & $\mathrm{H} \cdots A$ & $D \cdots A$ & $D-\mathrm{H} \cdots A$ \\
\hline $\mathrm{N} 1-\mathrm{H} 1 B \cdots \mathrm{O} 5 A^{\mathrm{i}}$ & $0.84(4)$ & $2.38(4)$ & $3.178(7)$ & $158(3)$ \\
$\mathrm{N} 1-\mathrm{H} 1 B \cdots \mathrm{O} 8 B^{\mathrm{i}}$ & $0.84(4)$ & $2.19(5)$ & $2.975(12)$ & $155(3)$ \\
$\mathrm{N} 1-\mathrm{H} 1 A \cdots \mathrm{O} 7 B^{\mathrm{ii}}$ & $0.91(3)$ & $2.06(4)$ & $2.885(9)$ & $150(3)$ \\
$\mathrm{N} 1-\mathrm{H} 1 A \cdots \mathrm{O} 7 A^{\mathrm{ii}}$ & $0.91(3)$ & $2.17(4)$ & $3.069(14)$ & $169(3)$ \\
$\mathrm{N} 1-\mathrm{H} 1 A \cdots \mathrm{O} 8 B^{\mathrm{ii}}$ & $0.91(3)$ & $2.35(4)$ & $3.086(13)$ & $138(3)$ \\
$\mathrm{N} 2-\mathrm{H} 2 A \cdots \mathrm{O} 2$ & $0.87(8)$ & $2.09(8)$ & $2.947(6)$ & $168(6)$ \\
$\mathrm{N} 2-\mathrm{H} 2 B \cdots \mathrm{O} 3^{i i i}$ & $0.92(6)$ & $2.04(6)$ & $2.951(6)$ & $171(5)$
\end{tabular}

Symmetry codes: (i) $x, y+1, z$; (ii) $-x+2,-y+1,-z$; (iii) $-x+1, y+1 / 2,-z-1 / 2$. 